\title{
Review Article \\ Overview of Cellular Transplantation in Diabetes Mellitus: Focus on the Metabolic Outcome
}

\author{
Livio Luzi, ${ }^{1,2}$ Stefano Benedini, ${ }^{1,2}$ Andrea Caumo, ${ }^{2}$ and Ileana Terruzzi ${ }^{3}$ \\ ${ }^{1}$ Metabolism Research Center and Endocrinology and Metabolism, IRCCS Policlinico San Donato Milanese, 20097 Milan, Italy \\ ${ }^{2}$ Department of Biomedical and Health Sciences, University of Milan, 20121 Milan, Italy \\ ${ }^{3}$ Diabetes Research Institute, San Raffaele Scientific Institute, 20132 Milan, Italy \\ Correspondence should be addressed to Livio Luzi; livio.luzi@unimi.it
}

Received 16 September 2014; Accepted 29 December 2014

Academic Editor: Sarantis Livadas

Copyright (C) 2015 Livio Luzi et al. This is an open access article distributed under the Creative Commons Attribution License, which permits unrestricted use, distribution, and reproduction in any medium, provided the original work is properly cited.

\begin{abstract}
Diabetes mellitus is a metabolic disease possible to treat via several different therapeutic approaches. Since the advent of insulin in 1922, type 1 diabetes mellitus has become a chronic treatable disease. Nonetheless, type 1 diabetes mellitus can be a devastating disease when the macro- and microangiopathic complications take place after several years of illness. Starting from the eighties, pancreas/islet transplantation has become a potential innovative treatment of diabetes mellitus. The major advantage of pancreas/islet transplantation is the restoration of c-peptide cosecretion along with insulin; the major disadvantage is the need to administer immunosuppressive drugs which are diabetogenic themselves. Islet transplantation is the progenitor of more recent forms of cellular and stem cell therapies which will be reviewed herein. Cellular therapies for diabetes mellitus are still an experimental procedure. Herein we present the actual current achievements and an outlook of close future possible advancements in the area of cellular transplantation for the cure of diabetes mellitus.
\end{abstract}

\section{Introduction}

Diabetes mellitus (with associated diseases and complications) is one of the major emergencies of the XXI century. In 2011 within a world population of 7.0 billion, there were 366 million of diabetics with 280 million of IGT (impaired glucose tolerance) [1]. It is estimated that in 2030 there will be approximately 910 million of diabetics in the world [1]. The huge number of diabetic patients makes it mandatory to find a definitive treatment/cure for the disease, for obvious health-related, social, and economic reasons. Most of affected patients have type 2 diabetes, which is a polygenic disease with about a 50\% environmental component and a strong link with obesity and metabolic syndrome. Type 1 diabetes, the most serious form of the disease, is determined by autoimmunity along with a predisposing genetic background and has enormously increased in the last 3 decades [2]. Both types of diabetes require life-long medical management and either oral or subcutaneous insulin injection treatment with a major economic impact.

At present, pancreas and islet-cell transplantation are the only potential cures for type 1 diabetes, eliminating the need for insulin administration when reaching full success. Nonetheless, long-term immunosuppression may produce several severe metabolic complications, such as glucose intolerance or even overt diabetes and hyperlipidemia. Concerning type 2 diabetes, several old [3] and novel [4] pharmacological treatments are available, although the most effective, less costly, and affordable treatment is still lifestyle intervention. Unfortunately compliance with life-style intervention approaches is extremely low.

The above considerations outline the importance of a novel, effective treatment to provide the cure for diabetes mellitus. In the present outlook paper we analyze the existing experience of cellular therapies for diabetes mellitus and discuss potential future scientific developments of cell treatments for diabetes.

\section{History, Rationale, and Criteria for Cellular Transplantation in Diabetes Mellitus}

2.1. History of Pancreas and Islet Transplantation. The first pancreatic transplantation was performed in 1966, when 
Kelly et al. performed the first human, whole-organ pancreatic transplantation for treatment of type 1 diabetes mellitus [5]. Much of the early work was performed by Sutherland and colleagues at the University of Minnesota. The 1980s assessed the beginning of a new phase in pancreas transplantation, mainly due to newer immunosuppressive drugs becoming available and increased clinical experience [6].

Almost in parallel, in 1967, Lacy's group described a novel collagenase-based method to isolate islets of Langerhans, paving the way for future in vitro and in vivo islet experiments [7]. Subsequent studies showed that transplanted islets could reverse diabetes in both rodents and nonhuman primates [8, 9]. The first successful trial of human islet allotransplantation resulting in long-term reversal of diabetes was performed in 1990 [10]. Yet, despite continued procedural improvements, only about $10 \%$ of islet recipients in the late 1990s achieved euglycemia (normal blood glucose). In 2000, Dr. Shapiro and colleagues published a report describing seven consecutive patients who achieved euglycemia following islet transplantation using a steroid-free protocol and large numbers of donor islets, referred to as the Edmonton protocol [11]. This protocol has been adapted by islet transplant centers around the world and has greatly increased islet transplant success.

2.2. The Double-Edge of Immunosuppression: Efficacy versus Metabolic Effects. Diabetes mellitus is a metabolic disease, and pancreatic and islet transplantation require immunosuppressive treatment to avoid rejection. Most immunosuppressive drugs are diabetogenic. Since the 1990s we were able to show that low-dose prednisone treatment (10 mg/day) as currently used after organ transplantation carries minor metabolic effects, mainly inducing insulin resistance [12]. The advent of cyclosporine constituted an initial advancement inducing less effect on insulin sensitivity and only some effects on insulin secretion. Nonetheless rapamycin constituted the real advancement in the field. It is now 25 years since the antiproliferative and immunosuppressive properties of rapamycin were first reported [13]. The latter effects were initially overshadowed by the former, and the agent was pursued more for its anticancer [14] than for its potential transplantation effects. It is structurally similar to FK506 (Tacrolimus), but, unlike the calcineurin inhibitors, it has no effect on interleukin-2 production $[15,16]$, despite clearly interfering with antigen-stimulated lymphocyte responses.

Rapamycin is a potent inhibitor of endothelial injury in rat aortic allografts. When added to full-dose cyclosporine it achieves low rejection rates, but it augments the nephrotoxicity and hyperlipidemia of cyclosporine. On the other hand, it allows discontinuation of calcineurin inhibitors in stable kidney and liver patients suffering from nephrotoxicity late post-transplant. The side-effect profile of hyperlipidemia, delay wound healing, and possibly liver effects are coming into focus, and ways of minimizing these problems are being introduced. The lessons learned include the need for early adequate blood levels, the lack of correlation between dose and drug exposure, and the potency that allows marked dose reductions in calcineurin inhibitors and steroids [17].
The avoidance of steroidal immunosuppressive drugs dramatically reduced the derangements of glucose metabolism. This was shown by our research group in other populations of organ transplant patients, such as liver [18] and kidney recipients [12]. Moreover, cyclosporine A is known both to impair insulin secretion and to decrease insulin action [19] and the nephrotoxicity is a major problem of this therapy [20]. Two recent reviews and meta-analyses summarize the complexity of the clinical complications of immunosuppressive treatments $[21,22]$.

2.3. C-Peptide and Diabetic Complications. Since 1997, Cpeptide, which is secreted in the same molar amount along with insulin and was considered functionless, became known as a biologically active molecule [23]. From 1997 till today, several scientific reports underlined the multifacet cellular actions of c-peptide, which behaves as a hormone although, up to date, it was not possible to discover any receptor specific for it [24]. In contrast, increasing evidence is mounting that at least some of the biological activities of C-peptide are mediated by cross-reactivity with the insulin receptor [25]. $\mathrm{C}$-peptide causes an increase of $\mathrm{Na}-\mathrm{K}$ ATPase pump which is responsible for delay and/or reversal of microangiopathic diabetic complications (Figure 1) [23, 26]. The potential effect of c-peptide in preventing diabetic complications was shadowed by many factors: first, patients with type 2 diabetes develop diabetic complications as much as patients with type 1 diabetes, in the presence of high circulating levels of cpeptide. A partial explanation of this is the possibility that, along with insulin resistance, also a form of "c-peptide resistance" would develop; second, the already mentioned failure in identifying a specific c-peptide receptor stands against a biological activity of c-peptide fully distinct from insulin; third, the effect of $\mathrm{C}$-peptide on diabetic macroangiopathy is even more controversial [25]. Marx et al. hypothesized a pathogenic effect of c-peptide in the development of the atherosclerotic plaque [25].

Taken all together, the present scientific evidence of coinjecting c-peptide along with insulin to prevent diabetic complications needs further investigation. Nonetheless, in case of confirmation of the biological role of c-peptide, cell transplantation will be theoretically a highly powerful treatment of diabetes mellitus.

2.4. Criteria for Patient Selection. The preexisting disease leading to organ transplantation is important in facilitating the de novo development of diabetes mellitus. The worst theoretical condition regards patients already diabetic prior to transplantation. This is the rule for pancreas-transplanted patients and is quite common for kidney-transplanted patients who receive the organ for end-stage diabetic nephropathy [12]. In case of a cadaveric donor, it is also fundamental to know as much as possible about the genetic background, medical history, and metabolic picture of the donor. The fact that the donor was obese, insulin resistant, or, in general, with the metabolic syndrome is a negative predictor for the metabolic outcome of either the organ or the cellular transplant. 


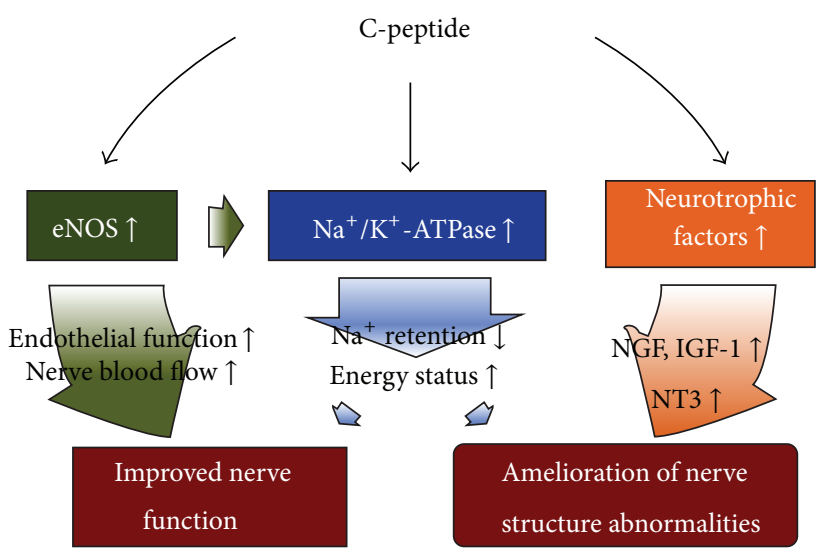

FIGURE 1: The three different intracellular mechanisms by which C-peptide can protect against diabetic complications and neuropathy, from [26, Figure 1, Page 505].

\section{Methodologies to Assess Metabolic and Nutritional Outcome}

3.1. Metabolic Outcome. We have recently shown that successful intraportal islet transplantation can normalize hepatic glucose production and insulin action in type 1 diabetic patients with a kidney transplant [27]. This procedure is relatively safe, noninvasive (percutaneous puncture of the liver), and repeatable, and it is performed in several centers worldwide $[28,29]$. The major factors limiting the largescale application of islet graft in diabetic patients receiving chronic immunosuppression for a kidney graft are (1) the low percentage of patients reaching insulin independence and a complete normalization of glucose homeostasis [28], (2) the limited number of organ donors available, and (3) the limited survival of fully successful grafts. In the long term, most diabetic patients receiving an islet graft achieve only partial function and a reduction of the pretransplant insulin requirement [30]; they are characterized by fasting Cpeptide concentrations in the near-normal range, although have abnormal fasting glucose and glycosylated hemoglobin. In the last decades, we [31-33] and others [34] found that protein and lipid metabolism had greater sensitivity to acutely infused insulin than did glucose metabolism, both in diabetes and in uremia. Those findings constituted the physiological bases to set the indication of performing pancreatic islet transplantation even if reaching only a partial function.

The isoglycemic, hyperinsulinemic clamp is aimed at studying the effect of insulin on glucose metabolism while glycaemia is maintained at the basal, pretest level by means of an exogenous glucose infusion, whereas the hyperglycemichyperinsulinemic clamp is considered the gold standard for the assessment of insulin secretion (Figure 2).

The HOMA index (HOMA stands for homeostatic model assessment) measuring insulin sensitivity (HOMA- IS) is calculated as follows: HOMA-IS $=22.5 /(\mathrm{gb} \times \mathrm{ib})$, where $\mathrm{gb}$ and $\mathrm{ib}$ are the basal glucose and insulin levels, respectively. The HOMA index measuring beta-cell secretion (HOMA-betacell $)$ is calculated as follows: HOMA-beta-cell $=20 \mathrm{ib} /(\mathrm{gb}$ - 3.5). Recently, the popularity of the HOMA indexes was justified by a number of validation studies and gained a large success as the most economical approach to measure at the same time insulin sensitivity and secretion [35]. Among the various dynamic tests that are available to the investigator, the oral glucose tolerance test has several advantages. It is very simple, it does not require trained personnel, the discomfort for the patient is minimized, and the perturbation is physiological. The results are so promising that it can be envisioned that in few years the OGTT will become the method for the simultaneous assessment of glucose tolerance, insulin secretion, and insulin sensitivity. As far as insulin secretion is concerned, one should bear in mind that during the OGTT the first- and second-phase insulin secretion are not clearly distinguishable. What can be determined are two components of insulin secretion, namely, the dynamic and the static components. The former component is related to the sensitivity of the beta-cell to the rate of change of glucose, while the latter component is related to the sensitivity of the beta-cell to the excursion of glucose.

An additional oral model (more physiological than the OGTT) is the mixed meal, which is able to asses both insulin sensitivity and beta-cell function (by disposition index) like the OGTT [36].

The IVGTT consists in rapidly injecting a bolus of glucose intravenously. The IVGTT allows the investigator to clearly distinguish the first-phase insulin secretion. In addition, if the glucose bolus is followed at $20 \mathrm{~min}$ by an insulin bolus, the insulin sensitivity of the subject under study can be determined from the minimal model analysis of the glucose and insulin data sets. The IVGTT is less labor-intensive than the OGTT. The advantage of the IVGTT over the OGTT is that the first-phase of insulin response is clearly visible despite being more physiological.

One specific issue is the determination of insulin sensitivity in patients with a quite low residual secretory rate. This is because the glucose stimulus may not elicit a high enough insulin secretory burst to calculate insulin sensitivity from the decay curve. For this reason the combination of regular insulin injection and IVGTT has been proposed to increase the insulin peak. Insofar no similar insulin administration 


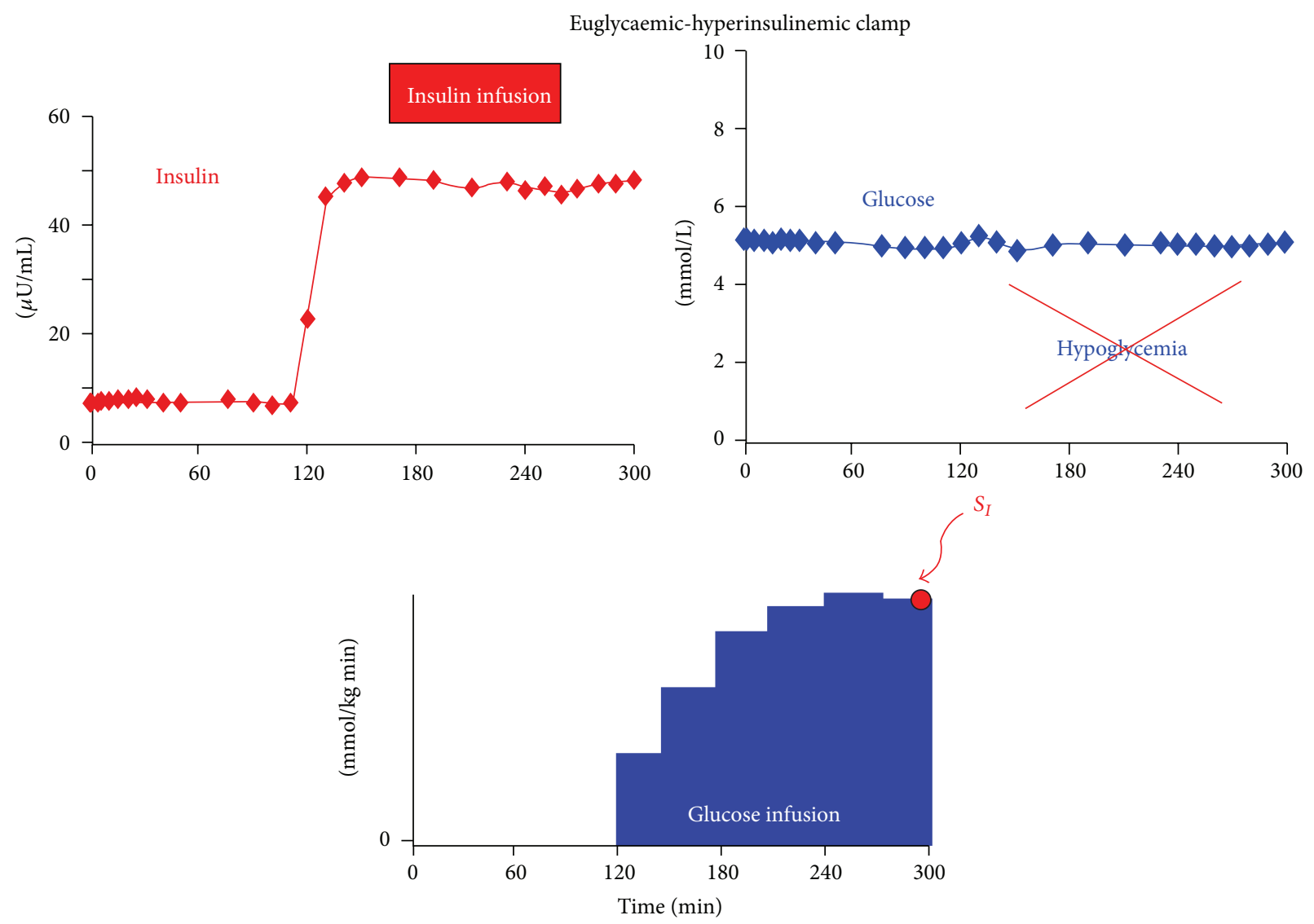

(a)

Hyperglycaemic-hyperinsulinemic clamp
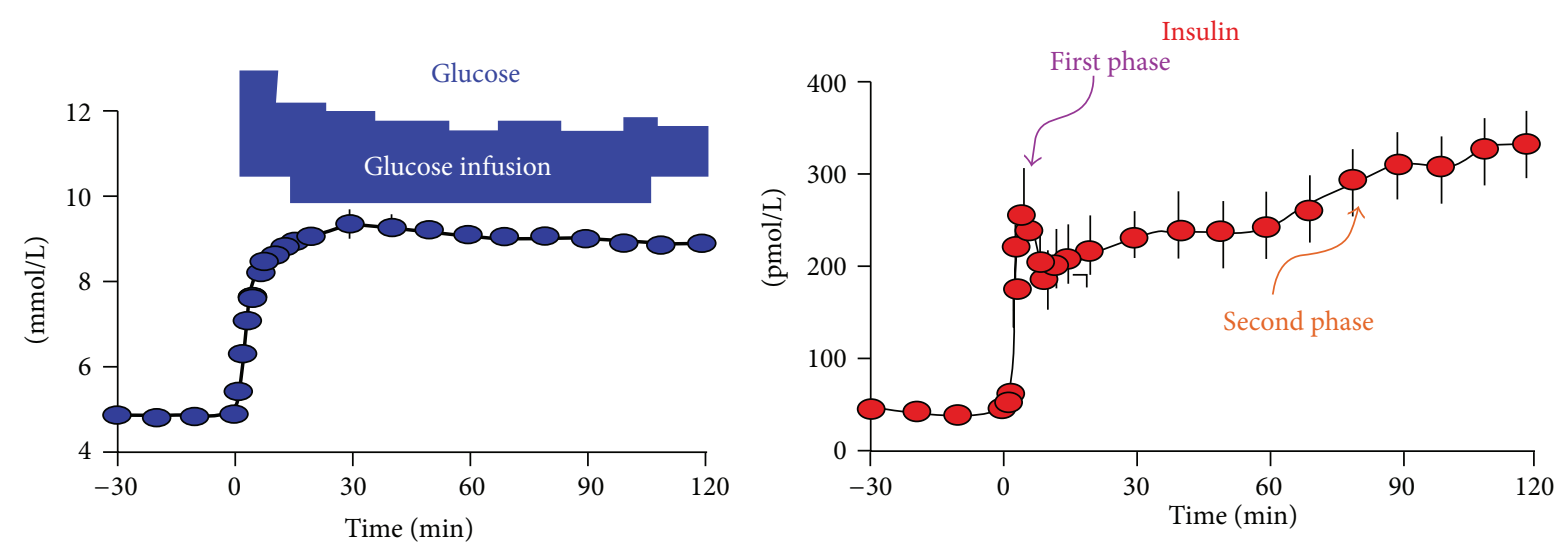

(b)

FIGURE 2: Schematic representation of euglycaemic-hyperinsulinemic clamp (a) and hyperglycaemic-hyperinsulinemic clamp (b) with graphic representation of glucose and insulin plasma concentrations, from [68, Figure 1, Page 157].

has been validated in conjunction with OGTT although a similar novel test would be advisable.

3.2. Nutritional Outcome. The prolonged success of the metabolic effects of islet transplantation raises the question on the nutritional effect of the cell transplantation procedure in type 1 diabetic patients and also in the light of the necessity to administer immunosuppressive drugs. In a tenyear follow-up we showed that islet transplantation caused an initial (1-3 years) decrease of body weight and BMI, accompanied by a decrease of waist circumference and carbohydrates intake, followed by a rebound increase of all nutritional parameters during years 7-10 after islet transplantation 


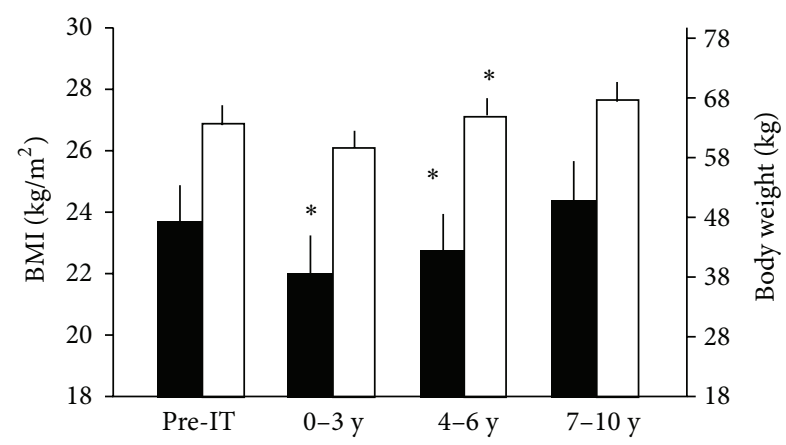

FIgURE 3: Body mass index (BMI, $\mathrm{kg} / \mathrm{m}^{2}$, black) and body weight (kg, white) means per eras during ten years after islet transplantation; ${ }^{*} P<0.05$. Reprinted from [37, Figure 1, Page 2026], Copyright (2013) by Elsevier Inc. with permission from Elsevier.

(Figure 3). The explanation for the data is in part an effect of immunosuppressive drugs and in part the free diet after $\beta$ cell function replacement [37]. In fact, among immunosuppressive drugs, prednisone [11,38] and rapamycin [39] are the ones most likely responsible for a potential nutritional damage, mainly acting on muscle/protein metabolism and accretion. Nonetheless, we showed that low-dose prednisone (up to $10 \mathrm{mg} / \mathrm{day}[40]$ ) and rapamycin at the immunosuppressive regimen $[41,42]$ do not impair protein metabolism after transplantation. The marked improvement of insulin secretory capacity after pancreatic islet transplantation may also balance the potential detrimental effects of immunosuppressive drugs.

\section{Novel Cellular Therapies and Technologies for Type 1 Diabetes Mellitus}

Besides pancreatic islet transplantation, which has been already in the clinic since two decades, stem cell therapies are the most promising innovative cellular treatment for diabetes mellitus nowadays [43]. Only a few tentative trials in human have been performed insofar. The rationale of using totipotent cells to treat and potentially cure diabetes mellitus can be summarized in 3 different strategies: (1) stem cell to reconstruct beta-cell secretory machinery; (2) stem cell to inhibit/abolish local immunological response and help pancreatic islet transplantation in autoimmune diabetes; (3) stem cells to treat diabetic complications.

(1) In a recent review, Dominguez-Bendala's group reported novel approaches to substitute failing endocrine pancreas. Among those, stem cell therapies and cellular reprogramming, which seek to regenerate the depleted $\beta$-cell compartment, are thoroughly discussed [44]. Pennarossa et al. [45] recently published an original approach where human skin fibroblasts, following a brief demethylation step, were reprogrammed to synthetize insulin and used to reverse hyperglycemia in rodents. Pennarossa's report is presented as a successful example of cellular reprogramming to produce insulin. Nonetheless, the environment to which reprogrammed islets are exposed is particularly hostile, with the presence of inflammatory response and of the autoimmune attack.

(2) For this reason it is fundamental to develop strategies overcoming the detrimental effect of autoantibodies. Figliuzzi et al. have recently analyzed an approach via which mesenchymal stem cell may help the success of pancreatic islet transplantation [46]. Several other approaches have been attempting to identify immune privileged sites, in order to minimize the role of autoimmunity [47, 48].

(3) Very recent stem cell based approaches are aimed at treating diabetic complications which cause the major social economical and quality of life burden to patients. Diabetes mellitus is very frequently complicated by macrovascular diseases. Therefore, stem cell treatment approaches have been developed to mitigate the morbidity and mortality following myocardial infarction and peripheral vascular disease $[49,50]$. As microvascular diabetic complications are concerned, stem cell treatment approaches have been attempted for all of them. In fact, endothelial progenitor cells were proposed for diabetic retinopathy [51]; similarly mesenchymal stem cells are under experimentation for diabetic polyneuropathy [52, 53]. Finally, stem cell approaches for diabetic nephropathy are under investigation [54]. A clear-cut potential advantage of stem cell replacement therapy when compared to standard pancreatic islet transplantation is the possibility to avoid immunosuppression. This will open a fully new area of application in type 2 diabetes mellitus [55].

Concerning novel strategies for cellular transplantation in diabetes mellitus, 2 additional points should be made as follows.

(1) The most common site for pancreatic islet transplantation has been the intraportal one insofar [27]. Insulin secreted intraportally is going to be cleaved by approximately $50 \%$ at the first pass and vice versa the choice of a peripheral site has a clear-cut metabolic advantage determined by the lack of first pass cleavage of insulin by the liver, with the consequence of the need of less islet mass to achieve the same peripheral insulin concentration.

(2) Cellular encapsulation technologies have the enormous advantage of isolating the cellular graft from the immune attack $[56,57]$. Unfortunately the metabolic success of the encapsulated graft has been rather poor insofar, both in animal models and in humans. The principal reason for the lack of success is that, besides the immune isolation, encapsulated grafts also cause a reduction of nutrient and oxygen supply into the cellular graft [44]. 


\section{Final Considerations and Perspectives}

Cellular transplantation in diabetes mellitus can be considered a highly innovative and still experimental procedure, which should be performed only by highly experienced centers worldwide. The clinical and research experience gained by previous treatments of diabetes will help in finding eventually a cure for this strikingly devastating disease.

Successful pancreas transplantation has already been shown to correct the metabolic abnormalities of diabetes $[12,58]$. In 1996 our work demonstrated that intraportal islet transplantation (a procedure associated with a much lower morbidity than pancreas transplantation) also normalized hepatic glucose production and improved tissue glucose utilization in a selected cohort of diabetic patients [59]. Moreover, a study conducted by our group demonstrated that two indices of insulin sensitivity (HOMA-IS and QUICKI) were reliable measures of insulin sensitivity in kidney-pancreastransplanted patients [60] as well as in epidemiological studies [61]. In type 2 diabetes, the loss of the early surge of insulin release, associated with insulin resistance, is an early and quite common defect that may have a pathogenic role in the development of postprandial hyperglycemia $[62,63]$.

Therefore, in any patient receiving cellular treatment of diabetes mellitus, it is fundamental to quantify insulin secretion and insulin sensitivity, as accurately as possible. This will allow the early detection of graft dysfunction and facilitate the management of transplanted patients as the optimization of the antirejection therapy is concerned. In this line, the need to reduce health costs has given remarkable impulse to the use of surrogate indexes of insulin sensitivity and secretion derived from the measurements of glycaemia and insulinemia obtained from a single blood sample drawn in the postabsorptive state. The indexes HOMA-IS and QUICKI (in comparison with isoglycemic, hyperinsulinemic clamp) are simple reliable tools to assess insulin sensitivity in clinical and epidemiologic investigations in many other clinical and epidemiological investigations like chronic liver disease before and after liver transplantation [63]. One potential confounding factor is that diabetic patients may be treated also with insulin, besides receiving cellular therapies. Wallace et al. showed that it is possible to use HOMA to assess insulin sensitivity in subjects treated with insulin, but it is imperative to ensure that samples are taken when glucose and insulin concentrations are in a steady state. Anyhow, the use of HOMA in subjects on insulin needs further validation [64]. If the investigator needs a more accurate quantification of insulin secretion and insulin sensitivity, dynamic tests are mandatory. Cobelli and associates demonstrated that both IVGTT and meal tolerance tests could be used to illustrate how these different approaches would provide complimentary yet differing insights regarding the regulation of beta-cell function in humans [65].

Any new attempt to perform cellular replacement therapy in diabetic patients should be accompanied by a careful expert-drive assessment of insulin secretion and action.

Most immunosuppressive drugs (if not all of them) have metabolic effects and impair insulin action [66], acting at different levels in the postinsulin receptor signaling cascade and the final effector metabolic pathways of carbohydrate, protein, and lipid/lipoprotein metabolism. Prednisone is well known to induce insulin resistance with respect to glucose metabolism [12]. Both cyclosporine and FK506 were shown by us to determine a modulation of insulin secretion and insulin action in liver-transplanted patients [67]. Rapamycin selectively inhibits the phosphorylation of p70 S6 kinase in vitro [39]. Alternative to immunosuppression are cellular transplantation strategies reducing the immune attack that are under development [46].

In perspective, the era of cellular replacement therapy of diabetes mellitus has already begun, although only the initial steps of a long run have been taken. Key clinical issues to improve the quality of life of diabetic patients will be (1) delaying the onset of diabetic complications, (2) avoiding the need of immunosuppressive drugs for their side-effects, and (3) prolonging life expectancy. Last but not the least, successful cellular transplantation has the potential of abating health costs of diabetes mellitus.

\section{Conflict of Interests}

The authors declare that there is no conflict of interests regarding the publication of this paper.

\section{Acknowledgment}

The authors wish to thank Mrs. Norma Trezzi for kindly supporting their research on type 1 diabetes.

\section{References}

[1] IDF, Diabetes Atlas, International Diabetes Federation, 6th edition, 2013.

[2] C. C. Patterson, G. G. Dahlquist, E. Gyürüs, A. Green, and G. Soltész, "Incidence trends for childhood type 1 diabetes in Europe during 1989-2003 and predicted new cases 2005-20: a Multicentre Prospective Registration Study," The Lancet, vol. 373, no. 9680, pp. 2027-2033, 2009.

[3] P. M. Thule and G. Umpierrez, "Sulfonylureas: a new look at old therapy topical collection on pharmacologic treatment of type 2 diabetes," Current Diabetes Reports, vol. 14, no. 4, article 473, 2014.

[4] N. Sattar, "Advances in managing type 2 diabetes: challenging old paradigms and developing new ones," F1000Prime Reports, vol. 6, article 42, 2014.

[5] W. D. Kelly, R. C. Lillehei, F. K. Merkel, Y. Idezuki, and F. G. Goetz, "Allotransplantation of the pancreas and duodenum along with the kidney in diabetic nephropathy," Surgery, vol. 61, no. 6, pp. 827-837, 1967.

[6] R. J. Stratta, "Review of immunosuppressive usage in pancreas transplantation," Clinical Transplantation, vol. 13, no. 1, pp. 1-12, 1999.

[7] P. E. Lacy and M. Kostianovsky, "Method for the isolation of intact islets of Langerhans from the rat pancreas," Diabetes, vol. 16, no. 1, pp. 35-39, 1967.

[8] P. E. Lacy, "Transplantation of islet cells-isografts and allografts," Monographs in pathology, vol. 21, pp. 156-165, 1980. 
[9] P. E. Lacy, J. M. Davie, and E. H. Finke, "Transplantation of insulin-producing tissue," The American Journal of Medicine, vol. 70, no. 3, pp. 589-594, 1981.

[10] D. W. Scharp, P. E. Lacy, J. V. Santiago et al., "Results of our first nine intraportal islet allografts in type 1, insulin-dependent diabetic patients," Transplantation, vol. 51, no. 1, pp. 76-85, 1991.

[11] A. M. J. Shapiro, C. Ricordi, B. J. Hering et al., "International trial of the Edmonton protocol for islet transplantation," The New England Journal of Medicine, vol. 355, no. 13, pp. 1318-1330, 2006.

[12] L. Luzi, A. Secchi, F. Facchini et al., "Reduction of insulin resistance by combined kidney-pancreas transplantation in Type 1 (insulin-dependent) diabetic patients," Diabetologia, vol. 33, no. 9, pp. 549-556, 1990.

[13] R. R. Martel, J. Klicius, and S. Galet, "Inhibition of the immune response by rapamycin, a new antifungal antibiotic," Canadian Journal of Physiology and Pharmacology, vol. 55, no. 1, pp. 48-51, 1977.

[14] J. Douros and M. Suffness, "New antitumor substances of natural origin," Cancer Treatment Reviews, vol. 8, no. 1, pp. 6387, 1981.

[15] F. J. Dumont, M. J. Staruch, S. L. Koprak et al., “The immunosuppressive and toxic effects of FK-506 are mechanistically related: pharmacology of a novel antagonist of FK-506 and rapamycin," The Journal of Experimental Medicine, vol. 176, no. 3, pp. 751-760, 1992.

[16] J. Liu, J. D. Farmer Jr., W. S. Lane, J. Friedman, I. Weissman, and S. L. Schreiber, "Calcineurin is a common target of cyclophilincyclosporin A and FKBP-FK506 complexes," Cell, vol. 66, no. 4, pp. 807-815, 1991.

[17] A. S. MacDonald, "Rapamycin in combination with cyclosporine or tacrolimus in liver, pancreas, and kidney transplantation," Transplantation Proceedings, vol. 35, no. 3, supplement, pp. S201-S208, 2003.

[18] G. Perseghin, E. Regalia, A. Battezzati et al., "Regulation of glucose homeostasis in humans with denervated livers," The Journal of Clinical Investigation, vol. 100, no. 4, pp. 931-941, 1997.

[19] S. L. Gillison, S. T. Bartlett, and D. L. Curry, "Synthesis-secretion coupling of insulin: effect of cyclosporin," Diabetes, vol. 38, no. 4, pp. 465-470, 1989.

[20] R. Shapiro, "Low toxicity immunosuppressive protocols in renal transplantation," The Keio Journal of Medicine, vol. 53, no. 1, pp. 18-22, 2004.

[21] W. H. Lim, J. Eris, J. Kanellis et al., "A systematic review of conversion from calcineurin inhibitor to mammalian target of rapamycin inhibitors for maintenance immunosuppression in kidney transplant recipients," The American Journal of Transplantation, vol. 14, no. 9, pp. 2106-2119, 2014.

[22] N. Murakami, L. V. Riella, and T. Funakoshi, "Risk of metabolic complications in kidney transplantation after conversion to mTOR inhibitor: a systematic review and meta-analysis," The American Journal of Transplantation, vol. 14, no. 10, pp. 23172327, 2014.

[23] Y. Ido, A. Vindigni, K. Chang et al., "Prevention of vascular and neural dysfunction in diabetic rats by C- peptide," Science, vol. 277, no. 5325, pp. 563-566, 1997.

[24] L. Luzi, G. Zerbini, and A. Caumo, "C-peptide: a redundant relative of insulin?” Diabetologia, vol. 50, no. 3, pp. 500-502, 2007.

[25] N. Marx, D. Walcher, C. Raichle et al., "C-peptide colocalizes with macrophages in early arteriosclerotic lesions of diabetic subjects and induces monocyte chemotaxis in vitro," Arteriosclerosis, Thrombosis, and Vascular Biology, vol. 24, no. 3, pp. 540-545, 2004.

[26] J. Wahren, K. Ekberg, and H. Jornvall, "C-peptide is a bioactive peptide," Diabetologia, vol. 50, no. 3, pp. 503-509, 2007.

[27] L. Luzi, G. Perseghin, M. D. Brendel et al., "Metabolic effects of restoring partial Beta-cell function after islet allotransplantation in type 1 diabetic patients," Diabetes, vol. 50, no. 2, pp. 277-282, 2001.

[28] B. J. Hering, C. C. Browatzki, A. Schultz, R. G. Bretzel, and K. F. Federlin, "Clinical islet transplantation-registry report, accomplishments in the past and future research needs," Cell Transplantation, vol. 2, no. 4, pp. 269-282, 1993, discussion 283305.

[29] R. Alejandro, D. H. Mintz, J. Noel et al., "Islet cell transplantation in type I diabetes mellitus," Transplantation Proceedings, vol. 19, no. 1, part 3, pp. 2359-2361, 1987.

[30] N. J. M. London, G. S. M. Robertson, D. R. Chadwick, P. R. V. Johnson, R. F. L. James, and P. R. F. Bell, "Human pancreatic islet isolation and transplantation," Clinical Transplantation, vol. 8, no. 5, pp. 421-459, 1994.

[31] L. Luzi, P. Castellino, D. C. Simonson, A. S. Petrides, and R. A. Defronzo, "Leucine metabolism in IDDM: role of insulin and substrate availability," Diabetes, vol. 39, no. 1, pp. 38-48, 1990.

[32] L. Luzi, A. S. Petrides, and R. A. de Fronzo, "Different sensitivity of glucose and amino acid metabolism to insulin in NIDDM," Diabetes, vol. 42, no. 12, pp. 1868-1877, 1993.

[33] L. Luzi, L. C. Groop, G. Perseghin et al., "Effect of pancreas transplantation on free fatty acid metabolism in uremic IDDM patients," Diabetes, vol. 45, no. 3, pp. 354-360, 1996.

[34] L. C. Groop, R. C. Bonadonna, S. DelPrato et al., "Glucose and free fatty acid metabolism in non-insulin-dependent diabetes mellitus. Evidence for multiple sites of insulin resistance," The Journal of Clinical Investigation, vol. 84, no. 1, pp. 205-213, 1989.

[35] D. R. Matthews, J. P. Hosker, A. S. Rudenski, B. A. Naylor, D. F. Treacher, and R. C. Turner, "Homeostasis model assessment: insulin resistance and Beta-cell function from fasting plasma glucose and insulin concentrations in man," Diabetologia, vol. 28, no. 7, pp. 412-419, 1985.

[36] C. Dalla Man, A. Caumo, R. Basu, R. Rizza, G. Toffolo, and C. Cobelli, "Minimal model estimation of glucose absorption and insulin sensitivity from oral test: validation with a tracer method," The American Journal of Physiology-Endocrinology and Metabolism, vol. 287, no. 4, pp. E637-E643, 2004.

[37] V. Delmonte, E. M. Peixoto, R. Poggioli et al., “Ten years' evaluation of diet, anthropometry, and physical exercise adherence after islet allotransplantation," Transplantation Proceedings, vol. 45, no. 5, pp. 2025-2028, 2013.

[38] L. Luzi, L. P. Sereni, A. Battezzati, A. Elli, J. P. Soulillou, and D. Cantarovich, "Metabolic effects of a corticosteroidfree immunosuppressive regimen in recipients of pancreatic transplant," Transplantation, vol. 75, no. 12, pp. 2018-2023, 2003.

[39] M. E. Patti, E. Brambilla, L. Luzi, E. J. Landaker, and C. R. Kahn, "Bidirectional modulation of insulin action by amino acids," The Journal of Clinical Investigation, vol. 101, no. 7, pp. 1519-1529, 1998.

[40] S. Benedini, E. Ruffini, I. Terruzzi, M. Mancuso, and L. Luzi, "Glucose and leucine metabolism in lung tranplanted patients on low dose of steroids for immunosuppressive therapy," Transplantation Proceedings, vol. 40, no. 5, pp. 1566-1571, 2008. 
[41] L. Luzi, G. Perseghin, P. Maffi et al., "Improved metabolic outcome of islet transplantation (ITx) in T1DM: the role of rapamycin," Diabetes, vol. 53, supplement 2, p. A454, 2004.

[42] G. Perseghin, G. Lattuada, M. Danna et al., "Effect of rapamycin (Rapa) and of isolated intra-portal islets transplantation (ITx) on hepatic triglyceride (IHF) content in humans: a longitudinal study," in Proceedings of the ADA 68th Scientific Sessions, p. 1947, San Francisco, Calif, USA, June 2008.

[43] Board on Health Sciences Policy, Board on Life Sciences, Division on Earth and Life Studies, Institute of Medicine, and National Academy of Sciences, Stem Cell Therapies: Opportunities for Ensuring the Quality and Safety of Clinical Offerings: Summary of a Joint Workshop, National Academies Press, Washington, DC, USA, 2014.

[44] G. Orlando, P. Gianello, M. Salvatori et al., "Cell replacement strategies aimed at reconstitution of the $\beta$-cell compartment in type 1 diabetes," Diabetes, vol. 63, no. 5, pp. 1433-1444, 2014.

[45] G. Pennarossa, S. Maffei, M. Campagnol, L. Tarantini, F. Gandolfi, and T. A. L. Brevini, "Brief demethylation step allows the conversion of adult human skin fibroblasts into insulinsecreting cells," Proceedings of the National Academy of Sciences of the United States of America, vol. 110, no. 22, pp. 8948-8953, 2013.

[46] M. Figliuzzi, B. Bonandrini, S. Silvani, and A. Remuzzi, "Mesenchymal stem cells help pancreatic islet transplantation to control type 1 diabetes," World Journal of Stem Cells, vol. 6, no. 2, pp. 163-172, 2014.

[47] G. P. Fadini, F. Ferraro, F. Quaini, T. Asahara, and P. Madeddu, "Concise review: diabetes, the bone marrow niche, and impaired vascular regeneration," Stem Cells Translational Medicine, vol. 3, no. 8, pp. 949-957, 2014.

[48] H. Wu and R. I. Mahato, "Mesenchymal stem cell-based therapy for type 1 diabetes," Discovery Medicine, vol. 17, no. 93, pp. 139143, 2014.

[49] K. Y. Howangyin and J. S. Silvestre, "Diabetes mellitus and ischemic diseases: molecular mechanisms of vascular repair dysfunction," Arteriosclerosis, Thrombosis, and Vascular Biology, vol. 34, no. 6, pp. 1126-1135, 2014.

[50] C. Gu, S. Huang, D. Gao et al., "Angiogenic effect of mesenchymal stem cells as a therapeutic target for enhancing diabetic wound Healing," The International Journal of Lower Extremity Wounds, vol. 13, no. 2, pp. 88-93, 2014.

[51] N. Lois, R. V. McCarter, C. O’Neill, R. J. Medina, and A. W. Stitt, "Endothelial progenitor cells in diabetic retinopath," Frontiers in Endocrinology, vol. 5, article 44, 2014.

[52] G. C. Davey, S. B. Patil, A. O'Loughlin, and T. O'Brien, "Mesenchymal stem cell-based treatment for microvascular and secondary complications of diabetes mellitus," Frontiers in Endocrinology, vol. 5, p. 86, 2014.

[53] H. Mizukami and S. Yagihashi, "Exploring a new therapy for diabetic polyneuropathy-the application of stem cell transplantation," Frontiers in Endocrinology, vol. 5, article 45, 2014.

[54] M. E. Ezquer, F. E. Ezquer, M. L. Arango-Rodríguez, and P. A. Conget, "MSC transplantation: a promising therapeutic strategy to manage the onset and progression of diabetic nephropathy," Biological Research, vol. 45, no. 3, pp. 289-296, 2012.

[55] Q. Tong, L. Duan, Z. Xu et al., "Improved insulin secretion following intrapancreatic UCB transplantation in patients with T2DM," The Journal of Clinical Endocrinology and Metabolism, vol. 98, no. 9, pp. E1501-E1504, 2013.

[56] R. Krishnan, M. Alexander, L. Robles, C. E. Foster, and J. R. T. Lakey, "Islet and stem cell encapsulation for clinical transplantation," The Review of Diabetic Studies, vol. 11, no. 1, pp. 84-101, 2014.

[57] S. Tezza, M. Ben Nasr, A. Vergani et al., "Novel immunological strategies for islet transplantation," Pharmacological Research, 2014.

[58] G. Perseghin, H. Hilden, E. Bianchi et al., "Normal sensitivity of FFA metabolism despite resistance of glucose metabolism to insulin after pancreas transplantation," Diabetes, vol. 43, p. 138a, 1993.

[59] L. Luzi, B. J. Hering, C. Socci et al., "Metabolic effects of successful intraportal islet transplantation in insulin-dependent diabetes mellitus," The Journal of Clinical Investigation, vol. 97, no. 11, pp. 2611-2618, 1996.

[60] G. Perseghin, A. Caumo, L. P. Sereni, A. Battezzati, and L. Luzi, "Fasting blood sample-based assessment of insulin sensitivity in kidney-pancreas-transplanted patients," Diabetes Care, vol. 25, no. 12, pp. 2207-2211, 2002.

[61] Y. Song and S. Liu, "Insulin sensitivity and insulin secretion determined by homeostasis model assessment and risk of diabetes in a multiethnic cohort of women: the Women's Health Initiative Observational Study," Diabetes Care, vol. 30, no. 11, pp. 1747-1752, 2007.

[62] S. del Prato, P. Marchetti, and R. C. Bonadonna, "Phasic insulin release and metabolic regulation in type 2 diabetes," Diabetes, vol. 51, supplement 1, pp. S109-S116, 2002.

[63] G. Perseghin, A. Caumo, V. Mazzaferro et al., "Assessment of insulin sensitivity based on a fasting blood sample in men with liver cirrhosis before and after liver transplantation," Transplantation, vol. 76, no. 4, pp. 697-702, 2003.

[64] T. M. Wallace, J. C. Levy, and D. R. Matthews, "Use and abuse of HOMA modeling," Diabetes Care, vol. 27, no. 6, pp. 1487-1495, 2004.

[65] C. Cobelli, G. M. Toffolo, C. D. Man et al., "Assessment of $\beta$-cell function in humans, simultaneously with insulin sensitivity and hepatic extraction, from intravenous and oral glucose tests," American Journal of Physiology-Endocrinology and Metabolism, vol. 293, no. 1, pp. E1-E15, 2007.

[66] W. Cai, L. Hu, and J. G. Foulkes, "Transcription-modulating drugs: mechanism and selectivity," Current Opinion in Biotechnology, vol. 7, no. 6, pp. 608-615, 1996.

[67] L. A. Fernandez, R. Lehmann, L. Luzi et al., "The effects of maintenance doses of FK506 versus cyclosporin A on glucose and lipid metabolism after orthotopic liver transplantation," Transplantation, vol. 68, no. 10, pp. 1532-1541, 1999.

[68] S. Benedini, A. Caumo, I. Terruzzi, and L. Luzi, "Immunosuppressive therapy in pancreas and islet transplant: need for simultaneous assessment of insulin sensitivity and secretion," Journal of Diabetes Mellitus, vol. 3, no. 3, pp. 156-160, 2013. 


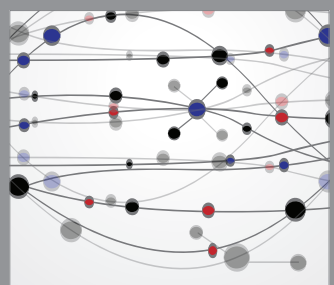

The Scientific World Journal
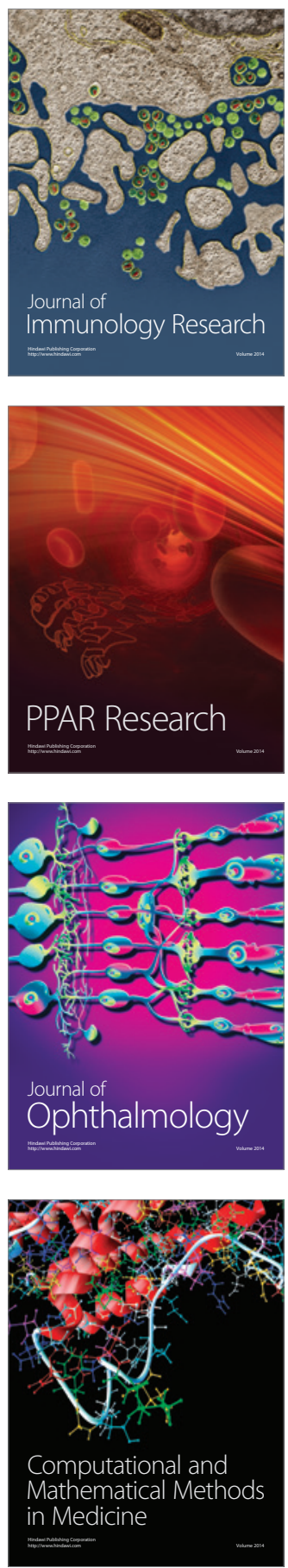

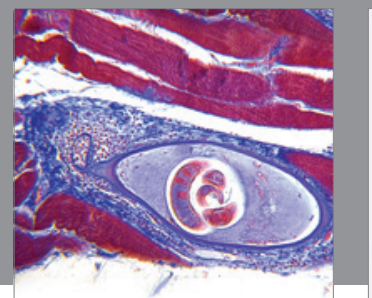

Gastroenterology

Research and Practice
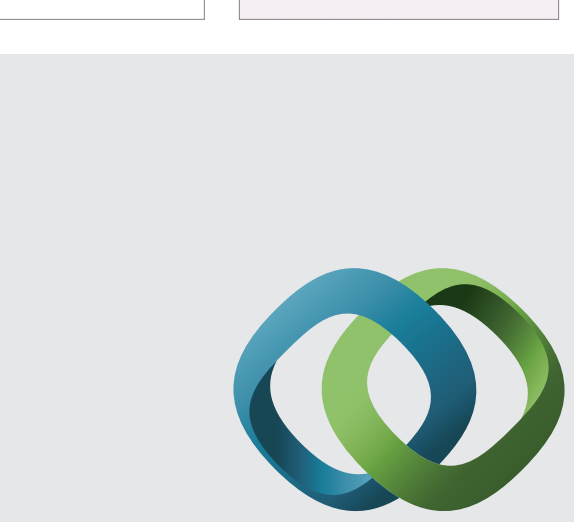

\section{Hindawi}

Submit your manuscripts at

http://www.hindawi.com
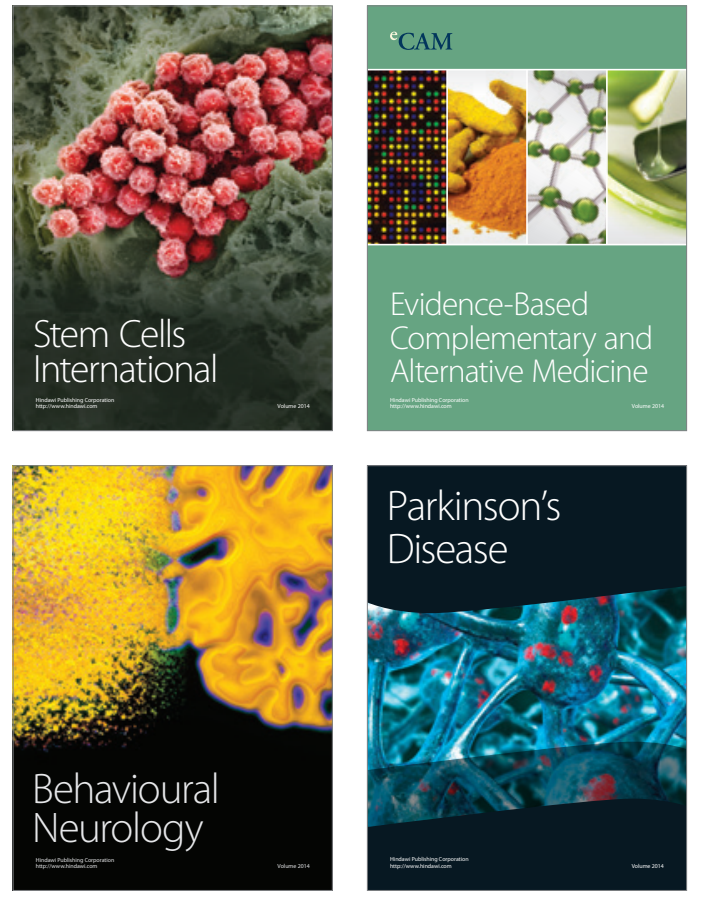
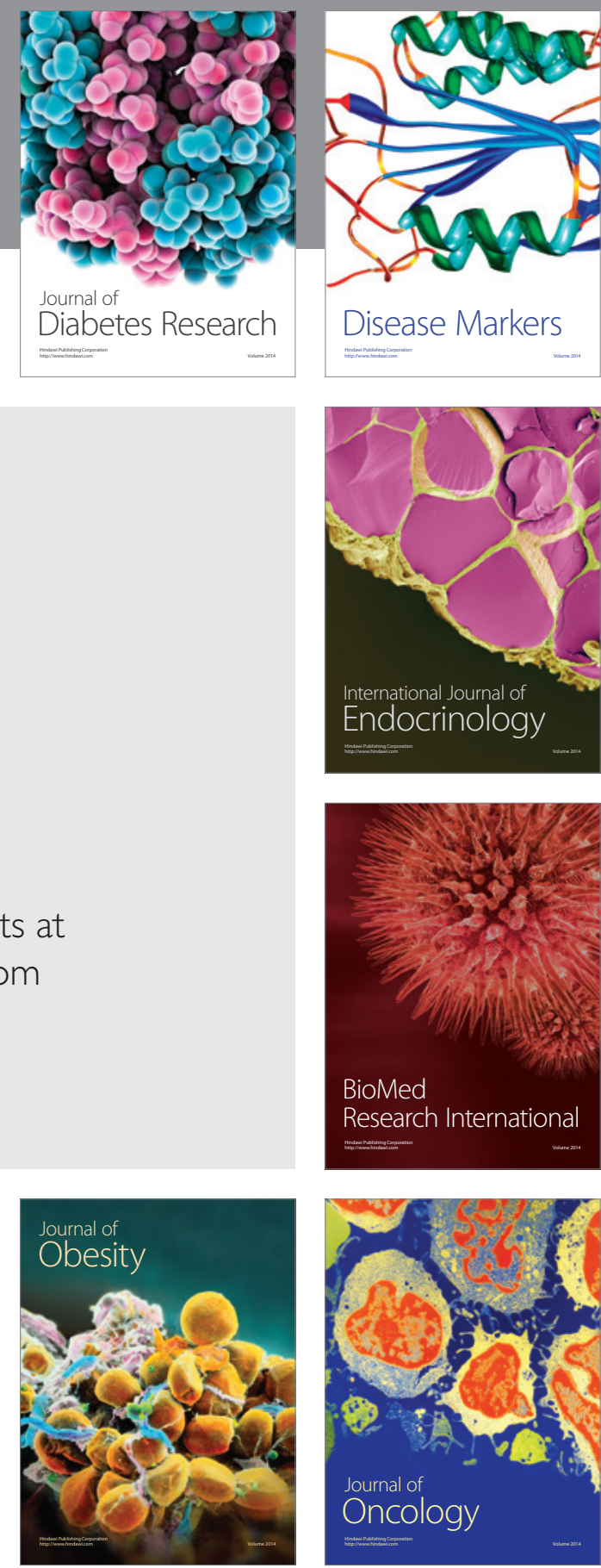

Disease Markers
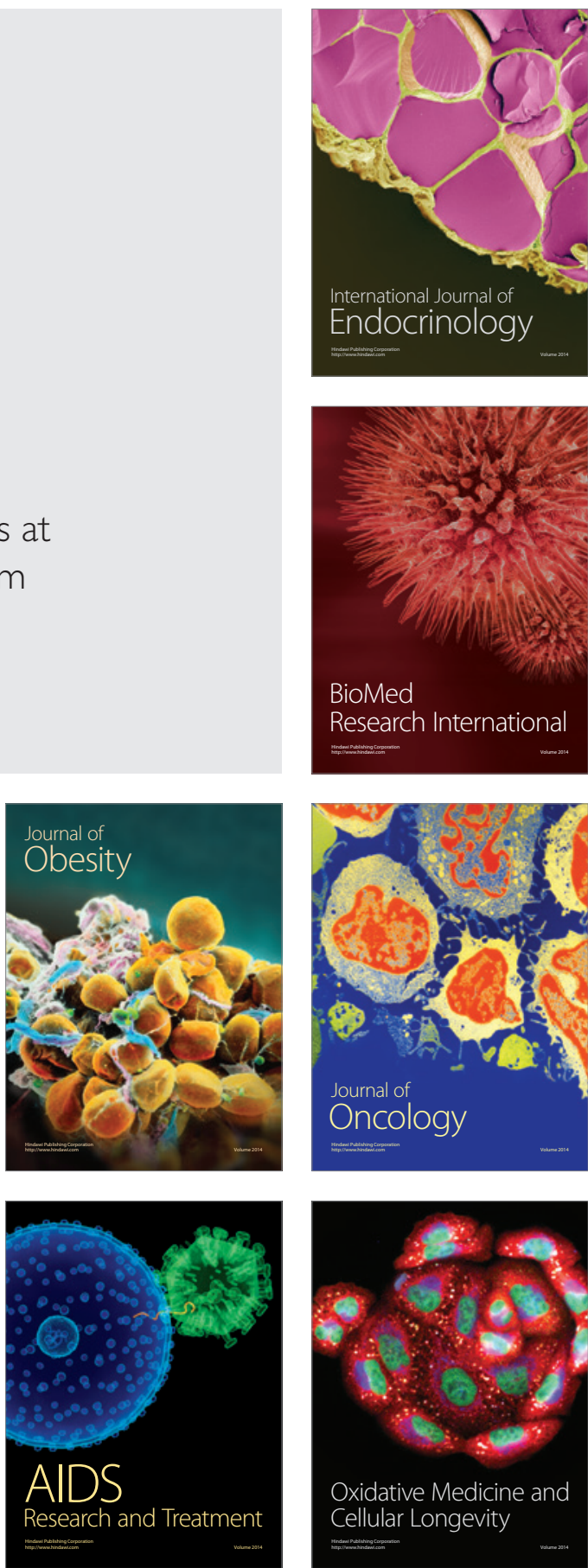